\title{
Sequential chemotherapy followed by radical thoracic radiotherapy (50 Gy in 25 fractions) in limited stage small cell lung cancer
}

\author{
Muhammad Shahid lqbal ${ }^{1}$, Joseph Carlow ${ }^{2}$, Fiona McDonald ${ }^{1}$, Philip Atherton ${ }^{1}$, Helen Turnbull ${ }^{1}$, Sandeep Singhal ${ }^{1}$, Timothy Simmons ${ }^{1}$, \\ Paula Mulvenna ${ }^{1}$, Josef Kovarik ${ }^{1}$, Rhona McMenemin ${ }^{3}$, Jill Gardiner ${ }^{4}$ and Alastair Greystoke ${ }^{4}$
}

${ }^{1}$ Department of Clinical Oncology, Northern Centre for Cancer Care, The Newcastle upon Tyne Hospitals NHS Foundation Trust, Newcastle upon Tyne, NE7 7DN, United Kingdom

${ }^{2}$ Newcastle University, Newcastle upon Tyne, United Kingdom

${ }^{3}$ Department of Clinical Oncology, Aberdeen Royal Infirmary, Aberdeen, AB25 2ZN, United Kingdom

${ }^{4}$ Department of Medical Oncology, Northern Centre for Cancer Care, The Newcastle upon Tyne Hospitals NHS Foundation Trust, Newcastle upon Tyne, NE7 7DN, United Kingdom

\section{Abstract}

Introduction: For limited stage small cell lung cancer (LS-SCLC) where concurrent chemoradiotherapy is inappropriate due to tumour bulk, co-morbidities or performance status, sequential treatment using chemotherapy followed by radiotherapy is the standard of care. The outcomes are not well established; we assessed in a single institution, the survival of these patients, prognostic factors and compared to the limited existing literature.

Materials and Method: Retrospective data was collected on all 59 patients diagnosed with LS-SCLC from 2011 to 2016 who received chemotherapy consisting of Carboplatin or Cisplatin + Etoposide followed by thoracic radiotherapy (50 Gy in 25 fractions) +/prophylactic cranial irradiation (PCI).

Results: Median age at diagnosis was 66 years (range 46-90). Patients received a median of four cycles of chemotherapy (range: $1-6$ ) and all the patients completed a full course of radiotherapy with only one patient experiencing grade $>2$ radiation induced toxicity. With a median follow up of 20.6 months, 45 patients had died with a median survival of 20.6 months. 2-year overall survival (OS) rates were $42 \%$. Age using a cut-off of 65 was prognostic (median OS 25.6 months $\leq 65$ years versus 14.1 months $>65$ years, $p=0.013$ ) but gender, stage and receipt of $\mathrm{PCl}$ were not.

Conclusions: Most of the literature reporting outcome from sequential treatment in LSSCLC is old and used a variety of radiotherapy regimens. Our data shows inferior outcomes to the gold standard concurrent chemoradiotherapy but support its usage in less fit patients with reasonable outcome observed.

Keywords: small cell lung carcinoma, sequential chemotherapy, thoracic radiotherapy

Correspondence to: Muhammad Shahid Iqbal Email:Shahid.lqbal@nhs.net

ecancer 2020, 14:1019

https://doi.org/10.3332/ecancer.2020.1019

Published: 09/03/2020

Received: 19/08/2019

Publication costs for this article were supported by ecancer (UK Charity number 1176307).

Copyright: $($ ) the authors; licensee ecancermedicalscience. This is an Open Access article distributed under the terms of the Creative Commons Attribution License (http:// creativecommons.org/licenses/by/3.0), which permits unrestricted use, distribution, and reproduction in any medium, provided the original work is properly cited. 


\section{Introduction}

Lung cancer is the third most common cancer amongst males and females in the UK [1] with 39,041 new cases diagnosed in 2016 [2]. Of these 39,041 cases small cell lung cancer makes up 10\%-15\% [2]. Small cell lung cancer (SCLC) typically presents at an advanced stage with two thirds of patients having distant metastases at the time of presentation [3], while SCLC should be staged according to the TNM (Tumour, Node, Metastases) classification; treatment may often be guided by whether the cancer is considered limited or extensive in stage using the US Veteran Association definition [4] where limited disease can be safely included in a radical radiotherapy field.

For limited stage small cell lung cancer (LS-SCLC) concurrent chemoradiotherapy (CCRT) is the standard of care, however, in patients who are deemed inappropriate for cCRT due to co-morbidities or tumour bulk, sequential therapy using chemotherapy followed by radical thoracic radiotherapy (TRT) can still result in cure and may be considered as a suitable treatment option. The research into LS-SCLC treated with sequential chemoradiotherapy (seqCRT) is sparse with multiple regimens reported.

The aim of this single-centre retrospective study is to analyse the survival of patients treated with sequential chemotherapy (cisplatin or carboplatin and etoposide) followed by TRT $50 \mathrm{~Gy}$ in 25 daily fractions, and the factors affecting the survival.

\section{Materials and methods}

Fifty nine consecutive patients, between September 2011 to August 2016 with biopsy proven LS-SCLC, treated with platinum and etoposide followed by TRT 50 Gy in 25 daily fractions were included. Data were collected retrospectively from patient records. All patients had staging investigations including computed tomography (CT) thorax abdomen and brain using TNM 7th edition, histological verification with either bronchoscopy \pm bronchial lavage, CT guided biopsy or endobronchial ultrasound, pulmonary lung function test, routine blood tests and ethylenediamine tetraacetic acid based glomerular function rate measurement. World Health Organisation (WHO) performance status (PS) was recorded for all patients.

\section{Chemotherapy}

Chemotherapy regimen consisted of 21 days cycle of combination of carboplatin (area under curve 5) on day 1 or cisplatin $75 \mathrm{mg} / \mathrm{m}^{2}$, with etoposide $120 \mathrm{mg} / \mathrm{m}^{2}$ intravenous infusion on day 1 followed by etoposide $100 \mathrm{mg}$ twice day orally on days 2 and 3 . The aim was to give four to six cycles of chemotherapy depending on tolerability, disease response and clinician's choice.

\section{Radiotherapy}

TRT was started three to four weeks after last cycle of chemotherapy. All patients underwent a contrast enhanced 3D CT scan for radiotherapy planning, comprising $3 \mathrm{~mm}$ slice thickness in supine position. A gross tumour volume (GTV) was drawn on each slice if there was residual/ visible disease post chemotherapy. A clinical tumour volume (CTV) comprised a 5-mm margin of GTV radiologically in all the directions. The aim was to cover post chemotherapy lung disease and pre-chemotherapy nodal disease in CTV by manual adjustment. Similar manual adjustment of CTV was permitted when disease adjacent to a structure such as a vertebra was not thought to invade the structure. Planning target volume (PTV) was then obtained using a further $10 \mathrm{~mm}$ margin craniocaudally and $8 \mathrm{~mm}$ laterally. The radiation dose of $50 \mathrm{~Gy}$ was prescribed to the isocentre and was delivered in 25 daily fractions.

For prophylactic cranial irradiation (PCI), thermoplastic immobilisation device was made for immobilisation and the target volume was whole brain. Two regimens, either $25 \mathrm{~Gy}$ in 10 daily fractions or $20 \mathrm{~Gy}$ in 5 daily fractions were used (depending on clinician's choice) using two lateral parallel opposed fields. 


\section{Statistics}

Overall survival was calculated from the date of diagnosis to either date of death or censoring on the date last seen. Statistical analysis was performed using SPSS (Version 24). Survival was calculated using the Kaplan-Meier method, with assessment of the differences in survival using log rank comparison and Cox regression analysis. The outcomes of interest were overall survival (OS) and progression free survival (PFS). Fisher's exact test was used to assess the association between use of $\mathrm{PCl}$ and the development of brain metastases. $\mathrm{A} p$ value of $<0.05$ was considered as statistically significant.

\section{Literature search}

A literature search was performed in Medline and EMBASE using the keywords sequential, late/delayed radiation, chemoradiotherapy, chemoradiation, small cell lung cancer, SCLC, limited stage. References of the studies identified were then searched for further studies to be included. Studies involving exclusive concurrent chemoradiotherapy were excluded.

\section{Ethics consideration}

This retrospective study was registered with the local hospital clinical effectiveness register as a service review project.

\section{Results}

The total number of patients who received treatment was 59. The median age at diagnosis was 66 years (range 46-90). The male to female ratio was 30:29. In 18 patients (30.5\%), CT-PET scan (positron emission tomography) was also available for staging. Fifty four patients (91\%) were stage III, 4 (7\%) were with stage II and 1 patient (2\%) was with stage I.

There were multiple reason(s) why patients did not receive CRT which have been grouped into five separate categories as shown in Table 1 with the most common being co-morbidities or poor performance status.

Patients received a median of four cycles of platinum-based doublet chemotherapy, carboplatin or cisplatin/etoposide (range 1-6). Two patients (3.4\%) received cisplatin instead of carboplatin. All 59 patients completed a subsequent full course of TRT, 50 Gy in 25 fractions. Radiotherapy was well tolerated with only one patient experienced grade III toxicity of pyrexia of unknown origin, hyponatraemia and confusion. Forty six patients (78\%) received $\mathrm{PCl}$. Four patients (7\%) declined $\mathrm{PCl}$, age greater than 75 was felt to be a contraindication in two patients and in one patient (2\%), $\mathrm{PCl}$ was not offered due to declining performance status. In the remaining six patients (10\%) who didn't receive $\mathrm{PCl}$, no reason was recorded.

Table 1. Reasons of not having concurrent chemoradiotherapy.

\begin{tabular}{|l|c|c|}
\hline \multicolumn{1}{|c|}{ Reason } & Number of patients & $\%$ \\
\hline Co-morbidities & 21 & 35.6 \\
\hline Poor performance status & 15 & 25.4 \\
\hline Too large tumour bulk & 10 & 16.9 \\
\hline Frailty & 5 & 8.5 \\
\hline Suspicious of metastases on baseline imaging* & 5 & 8.5 \\
\hline Not stated & 2 & 3.4 \\
\hline Patient declined & 1 & 1.7 \\
\hline
\end{tabular}

*Lesions not felt to be malignant on imaging post chemotherapy. 
With a median follow up of 20.6 months, 45 patients (76\%) had died and disease had recurred in 33 patients (56\%). In 25 cases ( $76 \%$ of the patients with disease recurrence), there were distant metastases and in remaining 8 patients (24\%), disease recurred locoregionally only. Of seven patients ( $21 \%$ of the relapsed patients) who developed brain metastases; four patients had received PCl whilst three had not received $\mathrm{PCI}(p=0.093)$. Twenty seven patients received further treatment, which included re-challenge platinum etoposide chemotherapy or oral topotecan therapy. None received radical re-irradiation.

Median PFS was 20.3 months (95\% Cl: 0-42.48, SE: 11.31) and was not significantly affected by gender $(p=0.268)$, age $(p=0.363)$, PS $(p=0.441)$, stage of disease $(p=0.571)$ or receipt of $\mathrm{PCl}(p=0.680)$ (Figure 1). The median overall survival OS was 20.6 months $(95 \% \mathrm{Cl}$ : 16.2-25.0) with a 1-year OS of $85 \%, 2$-year OS was $42 \%$ and 3-year OS of $32 \%$. Similarly, gender $(p=0.945)$, PS $(p=0.746)$, disease stage $(p=0.550)$ and $\mathrm{PCl}$ versus no $\mathrm{PCl}(p=0.623)$ (Figure 3$)$ didn't impact on OS. The only significant factor for survival was age. For patients $\leq 65$ years $(n=27)$, median OS was 25.6 months $(95 \% \mathrm{Cl}: 18.1-33.1)$ versus 14.1 months $(95 \% \mathrm{Cl}: 12.2-16.0)$ for patients $(n=32)>65$ years $(p=0.013$, Hazard Ratio 0.473, (95\% Cl: 0.26-0.85)) (Table 2, Figure 2).

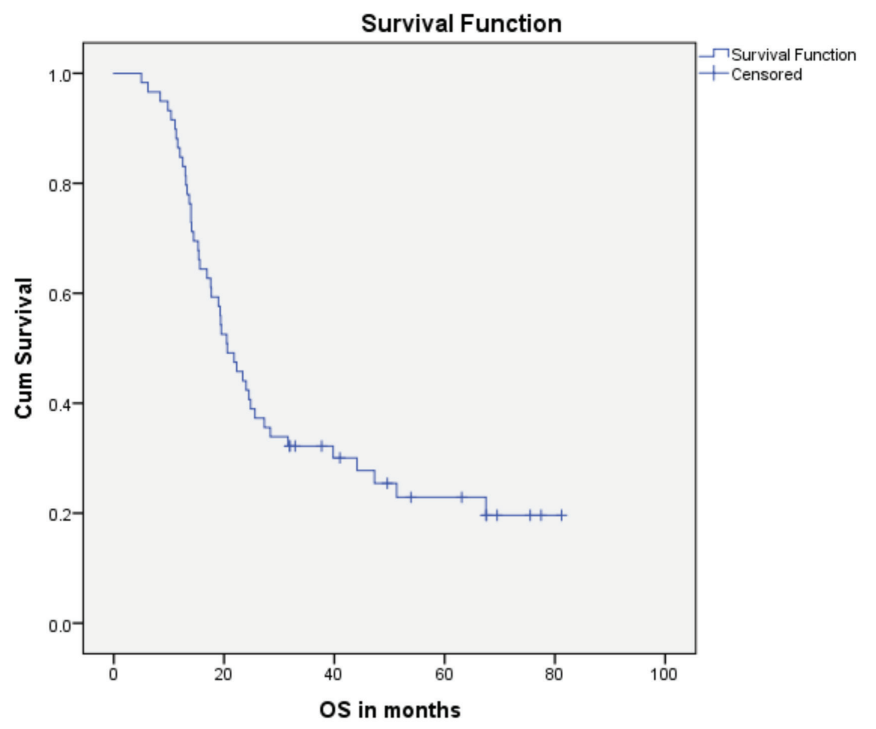

Figure 1. Kaplan-Meier curve for overall survival (in months).

Table 2. Prognostic factors affecting the overall survival demonstrated as median with $95 \%$ confidence intervals.

\begin{tabular}{|c|c|c|c|}
\hline $\begin{array}{c}\text { Characteristic } \\
n\end{array}$ & $\begin{array}{l}\text { Numbers } \\
59\end{array}$ & Results & $p$ value \\
\hline Male:female & $30: 29$ & $\begin{array}{l}\text { Male: } 20.5 \text { months }(95 \% \mathrm{Cl} 14.32-26.67) \\
\text { Female: } 20.6 \text { months }(95 \% \mathrm{Cl} 12.33-28.86)\end{array}$ & 0.94 \\
\hline Age $<65$ versus $\geq 65$ years & 27 versus 32 & $\begin{array}{l}\text { Age } \leq 65: 25.6 \text { months }(95 \% \mathrm{Cl} 18.13-33.06) \\
\text { Age }>65: 14.1 \text { months }(95 \% \mathrm{Cl} 12.21-15.98)\end{array}$ & 0.013 \\
\hline PS 0-1: 2-4 & 35 versus 24 & $\begin{array}{l}\text { PS } 0 \text { - } 119.3 \text { months (95\% Cl 11.76-26.83) } \\
\text { PS } 2 \text { - 4: } 20.6 \text { months ( } 95 \% \text { Cl 15.19-26.00) }\end{array}$ & 0.74 \\
\hline Stage I-IIla versus IIIb & 15 versus 44 & $\begin{array}{l}\text { Stage I-IIla: } 19.4 \text { months (95\%C 13.84-24.95) Stage IIIb: } 20.6 \text { month } \\
(95 \% \mathrm{Cl} 13.77-27.42)\end{array}$ & 0.55 \\
\hline PCI No vs Yes & 13 versus 46 & $\begin{array}{l}\text { No PCl: } 15.6 \text { months ( } 95 \% \mathrm{Cl} 1.74-29.45) \\
\text { Received PCl: } 20.6 \text { months (95\% Cl 16.16-25.03) }\end{array}$ & 0.62 \\
\hline
\end{tabular}




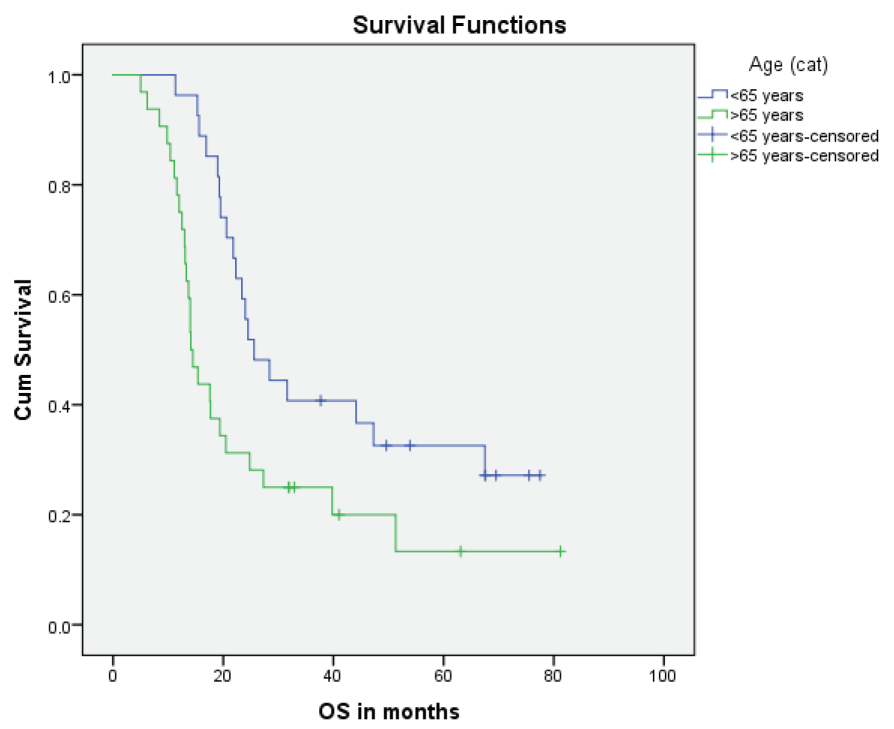

Figure 2. Kaplan-Meier curve for overall survival (in months) for patients $<65$ years versus $\geq 65$ years.

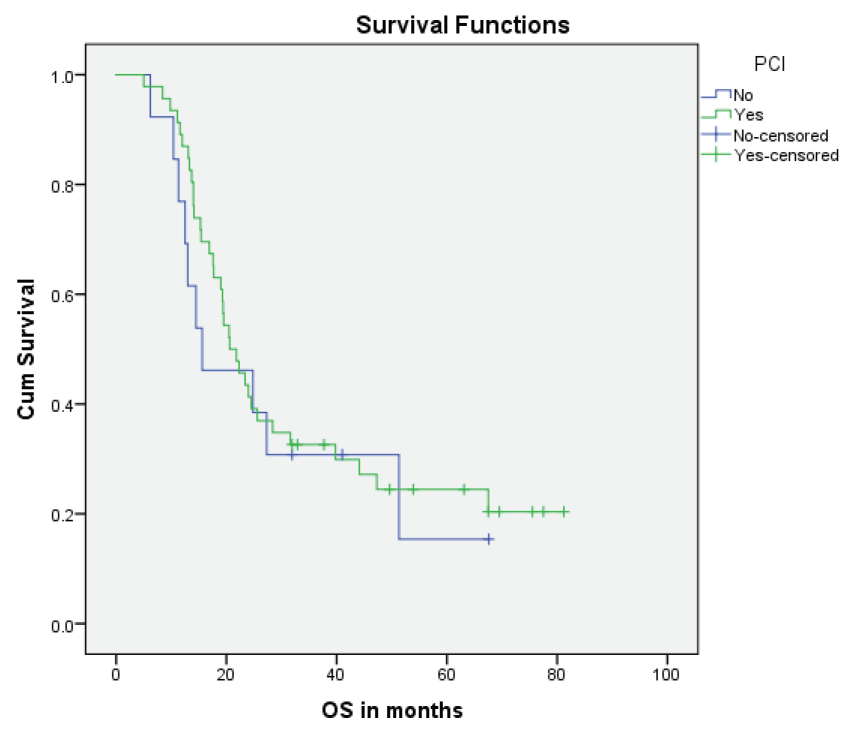

Figure 3. Kaplan-Meier curve for overall survival (in months) for patients who received PCI versus patients who didn't.

\section{Literature search}

\section{Sequential chemoradiotherapy (seqCRT) compared to concurrent chemoradiotherapy (cCRT)}

Two studies focused on comparing the difference in patients with LS-SCLC treated with either cCRT or sequential chemotherapy followed by TRT. In a phase III trial published in 2002 by Takada et al [5], the authors reported outcomes in patients with LS-SCLC treated with either 
cCRT or seqCRT. The study featured a large sample size of 231 patients who were randomised into the two groups. It showed improved survival (median OS of 27.2 months versus 19.7 months) with CCRT ( $p=0.097)$. In another randomised control trial published in 1997 by Gregor et al [6], 335 patients were randomised between arms (sequential and concurrent) of the study. It was found that those receiving cCRT had a median survival of 14 months compared to 15 months for patients receiving seqCRT.

\section{SeqCRT compared to chemotherapy alone}

Six studies were identified that investigated the impact of the addition of seqCRT versus chemotherapy alone. In 1988, Creech et al [7] reported results of a randomised control trial examining the benefit of sequential chemotherapy followed by TRT, 50 Gy in 25 fractions compared to chemotherapy alone. There was a significant survival advantage of having TRT (median OS 18.65 months versus 15.48 months; $p=0.003)$.

A retrospective study conducted by Lester et al [8] yielded similar results, with improved survival in patients who received thoracic radiotherapy over chemotherapy alone. The median survival for those receiving chemotherapy alone was 9.0 months compared to 17.7 months for those receiving seqCRT $(p<0.001)$. Rosenthal et al [9] also examined the impact of adding TRT to the survival of patients with LS-SCLC. It was found that the median OS with chemotherapy alone was 14 months compared to 18 months with seqCRT ( $p=0.073)$. Additionally, another study by Souhami et al [10] found a marginal improvement of a median survival (14.2 months with seqCRT versus 13.0 months receiving chemotherapy only) but this difference was non-significant. Similar findings were demonstrated by Kies et al [11] with a non-significant improvement in OS for those receiving seqCRT compared to the control group receiving chemotherapy alone. However, 38 of 42 patients who did not receive TRT had local relapse as compared to 20 of the 36 patients who did receive TRT.

Contrary to the above findings, one study showed no benefit of adding thoracic radiotherapy. Lebeau et al [12] reported that the median survival for chemotherapy alone was 496 days compared to a median survival of 316 days for sequential radiotherapy, however, this result was not statistically significant $(p=0.66)$. Similarly, in a small randomised study by Carlson et al (1991) [13], 24 patients received seqCRT. There was no significant difference in survival between those patients who received or didn't receive TRT. However, addition of TRT did reduce the incidence of local recurrences ( $29 \%$ versus $58 \% ; p=0.042$ ).

Two meta-analysis published in 1992 analysed the role of addition of TRT in LS-SCLC. Warde et al [14] showed a modest 2-year OS benefit (20\% with TRT as compared to $15 \%$ without TRT; the odds ratio was 1.53 with $95 \% \mathrm{Cl}: 1.30-1.76, p<.001$ ). A second meta-analysis published by Pignon et al [15] showed that the addition of TRT reduced the risk of mortality by $14 \%$ (Hazard ratio 0.86 with $95 \%$ Cl: $0.78-0.94$, $p=0.001)$. Three-year OS benefit was $5.4 \pm 1.4 \%$.

\section{SeqCRT-optimal radiation dose fractionation}

Various radiation dose fractionations, ranging from 32 to 50 Gy were used in the studies. From the studies included, 10 provided data on the median survival. It is clear from the literature that multiple dose fractionation regimens of sequential TRT exist within current practice. From the ten studies included eight different regimes were used and have been listed in Table 3 (in comparison to the current study).

\section{Radiotherapy related toxicities}

\section{Pulmonary toxicity}

Three studies highlighted pulmonary side effects amongst their patients receiving sequential TRT. Souhami et al [10] found that $35 \%$ of these patients experienced radiation induced pulmonary fibrosis with $40 \%$ of these patients having clinically evident dyspnoea. Additionally Ohnoshi et al [16] found that two patients died of radiation pneumonitis in the sequential arm of their trial. Similar results were obtained by Lebeau et al [12] who found that three patients had pneumonitis with one patient being clinical dyspnoeic as a result (4\%). 
Table 3. Dose fractionation used in studies using thoracic radiotherapy in limited stage small cell lung cancer with median overall survival achieved.

\begin{tabular}{|l|l|c|}
\hline \multicolumn{1}{|c|}{ Radiotherapy regimen } & \multicolumn{1}{c|}{ Studies used in } & \multicolumn{1}{c|}{ Median OS (months) } \\
\hline 50 Gy in 25 fractions & Creech et al (1981-85) [7] & 18.65 \\
\hline 50 Gy in 20 fractions & Gregor et al (1989-95) [6] & 15.00 \\
\hline 48 Gy in 22 fractions & Kies et al (1980-83) [11] & 20.00 \\
\hline 45 Gy in 30 fractions & Takada et al (2002) [5] & 15.74 \\
\hline & $\begin{array}{l}\text { Ohnoshi et al (1981-86) [16] } \\
\text { Souhami et al (1979-82) [10] } \\
\text { Ry in } 20 \text { fractions }\end{array}$ & \\
\hline 40 Gy in15 fractions & Lester et al (2000-2002) [8] & 17.70 \\
\hline 32 Gy in 8 fractions & Lebeau et al (1986-88) [12] & 11.29 \\
\hline 55 Gy in 30 fractions & Carlson et al (1991) [13] & 11.60 \\
\hline 50 Gy in 25 fractions & Current study & 20.60 \\
\hline
\end{tabular}

\section{Oesophageal toxicity}

Three studies reported patients experiencing oesophagitis. Takada et al [5] found that $4 \%$ of patient receiving seqCRT experienced severe oesophagitis (compared to $9 \%$ in cCRT arm). This was also found in Lebeau [12] with ten cases of severe oesophagitis reported in the seqCRT arm of the study and also by Souhami et al [10] who found that oesophagitis was a common side effect amongst those receiving sequential radiotherapy.

\section{Discussion}

A systematic review by Fried et al [17] included both cCRT and seqCRT found a significantly increased survival at 2 years for early TRT versus late TRT (HR 1.17 (95\% Cl: 1.02-1.35, $p=0.03$ ). Since the publication of Turisi et al study [18], cCRT with twice daily radiotherapy has become the standard of care in LS-SCLC.

For patients who are deemed unfit for CCRT, addition of sequential TRT to chemotherapy is a feasible option with literature suggesting a modest survival benefit but significantly better local control with acceptable toxicity. The majority of the relevant studies are from the 20 th century and used old radiotherapy techniques and the optimal TRT dose fractionation remains unknown. Modern imaging techniques have led to stage migration where some patients with extensive stage would previously have been classified as limited stage. Furthermore, with modern radiotherapy techniques it is possible to treat larger fields radically.

Our study demonstrated median survival of 20.3 months compared to 30 months (95\% Cl: 24-34) in twice daily concurrent chemoradiotherapy arm of a recently reported study (CONVERT) [19]. Similarly the two year survival was $42 \%$ in our series compared to 56\% (95\% Cl: $50 \%-62 \%$ ) in CONVERT study. Whilst inferior, seqCRT remains an option in patients who seemed unfit for cCRT and can result in reasonable survival in this patient population. In our series, age was associated with an adverse outcome using 65 years as cut off. This is in contrast to a second analysis of CONVERT study which showed no impact of age using cut of 70 years [20].

The treatment paradigm for extensive stage SCLC has changed with the use of atezolizumab in combination of carboplatin and etoposide in the first line setting [21]. TRT was not allowed in this study. Ongoing studies are assessing the potential impact of adding checkpoint inhibitors to concurrent chemoradiotherpay. Studies to investigate the potential benefit of checkpoint inhibitors in less fit patients receiving seqCRT should also be considered. 


\section{Conclusion}

The literature surrounding the use of sequential chemoradiotherapy is sparse and mostly published in the last century using old fashioned radiotherapy techniques with a range of dose and schedules. Our data showed inferior outcomes to the convert trial, which was performed in a similar time scale, as expected in this less fit patient population. However, it demonstrates reasonable outcomes and may be a valid treatment options in patients not suitable for concurrent treatment.

\section{Conflict of interest}

None declared.

\section{Funding}

No funding was received for this research study.

\section{References}

1. Cancer research UK. Lung cancer statistics. [https://www.cancerresearchuk.org/health-professional/cancer-statistics/statistics-bycancer-type/lung-cancer\#heading-Zero] Date accessed: 19/01/2019

2. Royal College of Physicians (2018). National lung cancer audit annual report 2017 (for the audit period of 2016) [Internet]. London [https://www.rcplondon.ac.uk/projects/outputs/nlca-annual-report-2017] Date accessed: 22/01/2019

3. BMJ Best Practice. Small cell lung cancer [https://bestpractice.bmj.com/topics/en-gb/1081/] Date accessed: 12/02/2019

4. Micke P, Faldum A, and Metz T, et al (2002) Staging small cell lung cancer: Veterans Administration Lung Study Group versus International Association for the Study of Lung Cancer--what limits limited disease? Lung Cancer 37(3) 271-276 https://doi.org/10.1016/ S0169-5002(02)00072-7 PMID: 12234695

5. Takada M, Fukuoka M, and Kawahara M, et al (2002) Phase III study of concurrent versus sequential thoracic radiotherapy in combination with cisplatin and etoposide for limited-stage small-cell lung cancer: results of the Japan Clinicial Oncology Group Study $9104 \mathrm{~J}$ Clin Oncol 20 3054-3060 https://doi.org/10.1200/JCO.2002.12.071 PMID: 12118018

6. Gregor Am Drings O, and Burghouts J, et al (1997) Randomised trial of alternating versus sequential radiotherapy/ Chemotherapy in limited-disease patients with small-cell lung cancer: a European Organisation for Research and Treatment of Cancer Lung Cancer Cooperative Group Study J Clin Oncol 15 2840-2849 https://doi.org/10.1200/JCO.1997.15.8.2840

7. Creech R, Richter M, and Finkelstein D (1988) Combination chemotherapy with or without consolidation radiation therapy (RT) for regional small cell carcinoma of the lung Proc Am Soc Clin Oncol 7 196. Abstract (ECOG [Eastern Cooperative Oncology Group] trial)

8. Lester JF, Hudson E, and Flubacher M, et al (2006) Small cell lung cancer treated in Southeast Wales. Cardiff 2006 Clin Oncol 18 378-382 https://doi.org/10.1016/j.clon.2006.03.016

9. Rosenthal MA, Tattersall MHN, and Fox RM, et al (1991) Adjuvant thoracic radiotherapy in small cell lung cancer: Ten-year follow-up of a randomised study Lung Cancer 7 235-241 https://doi.org/10.1016/0169-5002(91)90278-E

10. Souhami RL, Geddes DM, and Spiro SG, et al (1984) Radiotherapy in small cell cancer of the lung treated with combination chemotherapy: a controlled trial BMJ 288 1643-1646 https://doi.org/10.1136/bmj.288.6431.1643 PMID: 6326932 PMCID: 1441467 
11. Kies MS, Mira JG, and Crowley JJ, et al (1987) Multimodal therapy for limited small-cell lung cancer: a randomised study of induction combination chemotherapy with or without thoracic radiation in complete responders; and with wide-field versus reduced field radiation in partial responders: a Southwest Oncology Group Study J Clin Oncol 5 592-600 https://doi.org/10.1200/JCO.1987.5.4.592 PMID: 3031226

12. Lebeau B, Chastang C, and Brechor JM (1991) Small cell lung cancer (SCLC): negative results of a randomised clinical trial on delayed thoracic radiotherapyu administerd to complete responders (CR) patients Lung Cancer 7 Suppl 94. Abstract

13. Carlson RW, Sikic BI, and Gandara DR, et al (1991) Late consolidative radiation therapy in the treatment of limited stage small cell lung cancer Cancer 68 948-958 PMID: 1655219

14. Warde $P$ and Payne D (1992) Does thoracic irradiation improve survival and local control in limited-stage small-cell carcinoma of the lung? A meta-analysis J Clin Oncol 10(6) 890-895 https://doi.org/10.1200/JCO.1992.10.6.890 PMID: 1316951

15. Pignon JP, Arriagada R, and Ihde DC, et al (1999) A meta-analysis of thoracic radiotherapy for small-cell lung cancer N Engl J Med 340 265-271

16. Ohnoshi T, Hiraki S, and Kimura I (1987) Randomised trial of chemotherapy alone or with chest irradiation in limited stage small cell lung cancer Cancer Chemotherapy: Challenges for the Future vol 2, eds K Kimura, K Ota, and RB Herberman, et al Proceedings of the second Nagoya International Symposium on Cancer Treatment, Nagoya, Japan, October 16-18, 1986 (Amsterdam: Excerpta Medica) pp $186-191$

17. Fried B, Morris D, and Hensing T, et al (2004) Systematic review evaluating the timing of thoracic radiation therapy in combined modality therapy for limited stage small-cell lung cancer J Clin Oncol 22 4785-4793 https://doi.org/10.1200/JCO.2004.01.178

18. Turrisi AT III, Kim K, and Blum R, et al (1999) Twice-daily compared with once-daily thoracic radiotherapy in limited small-cell lung cancer treated concurrently with cisplatin and etoposide NEJM 340(4) 265-271 https://doi.org/10.1056/NEJM199901283400403 PMID: 9920950

19. Faivre-Finn C, Snee M, and Ashcroft L, et al (2017) Concurrent once-daily versus twice-daily chemoradiotherapy in patients with limited-stage small-cell lung cancer (CONVERT): an open-label, phase 3, randomised, superiority trial Lancet Oncol 18(8) 1116-1125 https://doi.org/10.1016/S1470-2045(17)30318-2 PMID: 28642008 PMCID: 5555437

20. Christodoulou M, Blackhall F, and Mistry H, et al (2019) Compliance and outcome of elderly patients treated in the concurrent once-daily versus twice-daily radiotherapy (CONVERT) trial J Thorac Oncol 14(1) 63-71 https://doi.org/10.1016/j.jtho.2018.09.027 PMCID: 6328625

21. Horn L, Mansfield AS, and Szczęsna A, et al (2018) First-line atezolizumab plus chemotherapy in extensive-stage small-cell lung cancer N Engl J Med 379(23) 2220-2229 PMID: 30280641 